\title{
Effects of Uremic Toxins on the Binding of Aripiprazole to Human Serum Albumin
}

\author{
Koji Nishi,${ }^{a}$ Keiki Sakurama, ${ }^{a}$ Hiroshi Watanabe, ${ }^{b}$ Toru Maruyama, ${ }^{b}$ Keishi Yamasaki, ${ }^{* a, c}$ and \\ Masaki Otagiri*,a,c \\ ${ }^{a}$ Faculty of Pharmaceutical Sciences, Sojo University; 4-22-1 Ikeda, Nishi-ku, Kumamoto 860-0082, Japan: \\ ${ }^{b}$ Graduate School of Pharmaceutical Sciences, Kumamoto University; 5-1 Oe-honmachi, Chuo-ku, Kumamoto 861- \\ 0973, Japan: and ${ }^{c}$ DDS Research Institute, Sojo University; 4-22-1 Ikeda, Nishi-ku, Kumamoto 860-0082, Japan. \\ Received November 18, 2020; accepted December 21, 2020
}

We recently reported that aripiprazole (ARP), an antipsychotic drug, binds strongly to human serum albumin (HSA), the major drug binding protein in serum. It is known that uremic toxins that accumulate during renal disease affect the interaction between HSA and drug binding. In this study, the issue of how uremic toxins (indoxyl sulfate, indole acetic acid and $p$-cresyl sulfate) affect the binding of ARP to HSA was investigated. Equilibrium dialysis experiments revealed that all uremic toxins inhibited the binding of ARP to HSA although the inhibitory effects differed, depending on the specific uremic toxin. The potency of inhibition can be partially explained by the affinities of uremic toxins to HSA. Fluorescence displacement experiments suggested that ARP as well as all uremic toxins bind to site II of HSA. The inhibitory effects of the toxins on the binding of ARP for the drugs binding to the diazepam subsite are significantly larger, comparing with those for binding to arylpropionic acids subsite. Interestingly, induced circular dichroism (CD) spectra indicated that the spatial orientation of $p$-cresyl sulfate in the binding pocket is different from that for indoxyl sulfate and indole acetic acid. The limited findings obtained herein are important data in considering the effects of uremic toxins on the pharmacokinetics of ARP and the drugs that bind to site II on HSA, particularly drugs binding to diazepam binding site in site II.

Key words human serum albumin; aripiprazole; uremic toxin; protein binding

\section{INTRODUCTION}

Human serum albumin (HSA), which is the most abundant in serum, primarily functions as a carrier of various exogenous and endogenous substances such as drugs, fatty acids, toxins and hormones. ${ }^{1)}$ A variety of drug molecules also bind to $\mathrm{HSA}^{2)}$ In the circulation, drug molecules are either bound to plasma proteins or are present as an unbound form. Unbound drug molecules, depending on their physico-chemical properties, can passively diffuse through physiological barriers. Thus, the binding of a drug to HSA controls the transfer of the free, active form of a drug in the circulation into tissue. Therefore, interactions of drug with HSA are important factors to evaluate the pharmacokinetics properties and interaction between drugs.

In renal and hepatic diseases, the binding of drugs to HSA are frequently altered. ${ }^{3,4)}$ It is widely accepted that the presence of accumulated endogenous inhibitors in the circulation affects the binding of a drug to HSA. Uremic toxins that are accumulated in the patients with kidney diseases also bind to HSA. ${ }^{5,6)}$ However, the issue of which uremic toxins inhibit drug-binding to HSA remains unclear. In fact, Ikeda et al. made the misleading conclusion that the binding of furosemide to bovine serum albumin is competitively inhibited by indoxyl sulfate, which is one of the typical uremic toxins. ${ }^{7)}$ To clarify this issue, systematic inhibitory experiments using uremic toxins that bind tightly to HSA should be done.

We recently reported that aripiprazole (ARP), an antipsychotic drug, shows high affinity binding to HSA. ${ }^{8,9)}$ An X-ray crystallographic study showed that the chlorine atom of ARP interacted with cysteine (Cys) residue at position 34 in HSA, similar to diazepam-HSA interactions. ${ }^{10)}$ Very recently, we reported the decreased binding affinity of ARP to chemically oxidized HSA as a model of HSA observed in the patients with chronic renal failure, ${ }^{11)}$ and indicated a significantly lower binding ability for oxidized HSA, compared to normal HSA. In our continuing investigations, the effects of uremic toxins on the binding of ARP to HSA were examined with the objective of obtaining basic information regarding ARP-HSA in renal diseased states.

\section{MATERIALS AND METHODS}

Materials Human serum albumin, essentially fatty acid free, was purchased from Sigma-Aldrich (Darmstadt, Germany). ARP and $p$-cresyl sulfate were obtained from Tokyo Chemical Industry Co., Ltd. (Tokyo, Japan). Indoxyl sulfate and indole acetic acid were purchased from Nacalai Tesque (Kyoto, Japan). All other chemicals and solvents were purchased from commercial sources and were of analytical grade.

Equilibrium Dialysis Equilibrium dialysis was performed using Sanko plastic dialysis cells (Fukuoka, Japan) at $25 \mathrm{bC}$ for $12 \mathrm{~h}$. According to our previous method, ${ }^{8)}$ same volume of the buffer and ARP-HSA mixture solutions were set to each compartment, respectively. After dialysis, the concentrations of ARP in the buffer ( $D f$, free ARP concentration) and HSA compartments ( $D t$, total concentrations of ligand) were measured by HPLC. The concentration of bound ARP to HSA $(D b)$ was calculated by subtracting $D f$ from $D t$. Free fractions were calculated using the values of $[D f]$ and $[D t]$ as follows, 


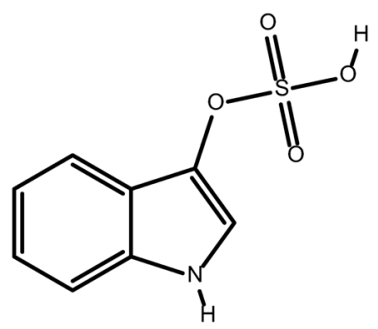

Indoxyl sulfate

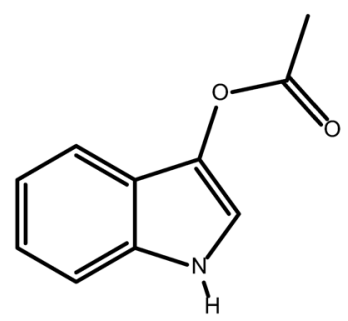

Indole acetic acid

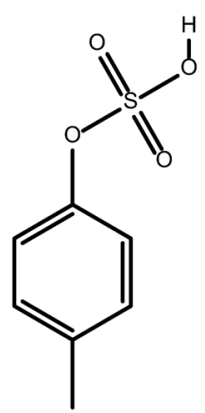

p-Cresyl sulfate

Fig. 1. Chemical Structures of Indoxyl Sulfate, Indole Acetic Acid and $p$-Cresyl Sulfate

$$
\text { Free fraction }=\frac{[D f]}{[D t]} \times 100
$$

where $D f$ is the concentration of the unbound drug, $D t$ is the total drug concentration and $D b$, the concentration of the bound drug, was calculated by subtracting $D f$, free drug concentration from the total drug concentration, $D t$. In equilibrium dialysis experiments, $20 \mu \mathrm{M}$ ARP and $40 \mu \mathrm{M}$ HSA, which are different from the concentrations in a clinical situation (ARP: approx. $0.1 \mu \mathrm{M}$ and HSA: approx. $600 \mu \mathrm{M}$ ) were used for the quantitative detection of ARP by HPLC.

Fluorescent Probe Displacement As fluorescent probes for site I and site II on HSA, warfarin and dansylsarcosine were used, respectively. Fluorescence spectra of probes were measured using a Hitachi F-2500 fluorescence spectrophotometer (Hitachi, Tokyo, Japan) at $25^{\circ} \mathrm{C}$. The excitation wavelengths for warfarin and dansylsarcosine were $320 \mathrm{~nm}$ and $350 \mathrm{~nm}$, respectively. The degree of displacement of these probes was calculated using the following equation,

$$
\text { Percentage of displacement }=\frac{\left(F_{1}-F_{2}\right)}{F_{1}} \times 100
$$

where $F_{1}$ and $F_{2}$ represent the maximum fluorescent intensity of the probe in HSA solution without and with displacers, respectively.

Circular Dichroism (CD) Spectra Measurements CD spectra were measured with a Jasco J-720 spectropolarimeter (Jasco, Tokyo, Japan), using a $10-\mathrm{mm}$ cell at $25^{\circ} \mathrm{C}$. The observed ellipticity $(\theta)$ was defined as the ellipticity of the uremic toxin-HSA mixed solution minus of HSA solution alone.

Statistical Analysis The overall differences between groups were determined with one-way of ANOVA.

\section{RESULTS}

Effect of Uremic Toxins on the Binding of ARP to HSA Accumulated uremic toxins in the circulation such as indoxyl sulfate, indole acetic acid and $p$-cresyl sulfate (Fig. 1) could displace a drug that is bound to HSA. To address this issue, we examined the effect of uremic toxins on the binding of ARP to HSA. In initial experiments, the effects of a mixture of uremic toxins at normal concentrations (indoxyl sulfate: $3 \mu \mathrm{M}$; indole acetic acid: $3 \mu \mathrm{M} ; p$-cresyl sulfate: $10 \mu \mathrm{M}){ }^{12)}$ on the binding of ARP to HSA were examined. The binding percentages of ARP to HSA in the presence or absence of solutions of mixtures of uremic toxins were $97.8 \pm 1.89$ or $98.3 \pm 1.78 \%$, respectively, suggesting that, under normal conditions, uremic toxins have no effect on ARP-HSA inter-

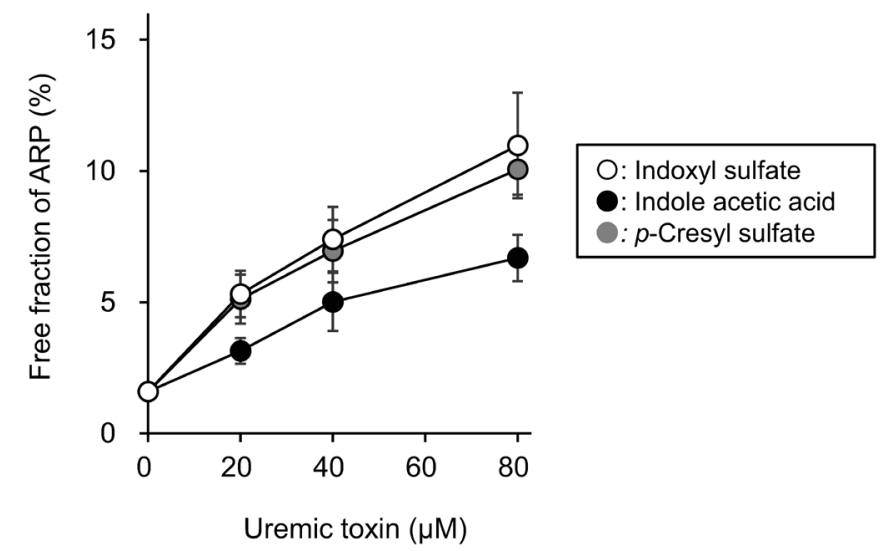

Fig. 2. Effect of Uremic Toxins on the Free Fraction of ARP at $\mathrm{pH} 7.4$ and $25^{\circ} \mathrm{C}$

The free fraction of ARP was measured in the presence of ARP $(20 \mu \mathrm{M})$, HSA $(40 \mu \mathrm{M})$ and each concentration $(0-80 \mu \mathrm{M})$ of uremic toxins. The values are presented as the mean \pm standard deviation (S.D.) of three independent experiments.

actions. The concentration dependency of uremic toxins on the inhibition of the binding of ARP to HSA was examined in subsequent experiments (Fig. 2). All investigated uremic toxins increased the free fractions of ARP and these occurred in a concentration-dependent manner (free fraction without uremic toxins: $1.60 \pm 0.11 \%$ ). It is particularly noteworthy that indoxyl sulfate and $p$-cresyl sulfate caused a marked increase in the free fraction of ARP. On the other hand, the effect of indole acetic acid on the binding of ARP was smaller than that for the two toxins. This difference can be attributed to differences in binding constants.

Binding Sites on HSA for Uremic Toxins We attempted to identify the binding sites for uremic toxins on HSA using typical fluorescent probes (site I; warfarin, site II; dansylsarcosine) (Fig. 3). When ARP and uremic toxins were present, the fluorescence of dansylsarcosine bound to HSA was significantly lower, and this decrease was dependent on the concentrations of the displacers. The effects of the displacers on reducing the fluorescence were in the order of ARP $>$ indoxyl sulfate $=p$-cresyl sulfate $>$ indole acetic acid. These results suggest that ARP and uremic toxins share the same binding site on HSA. The order of inhibitory effects by these three uremic toxins is basically the same tendency as the values for the binding constants of uremic toxin-HSA complexes (see Table 1). Only a slight change in the fluorescence of warfarin, which can be attributed to allosteric interactions, was observed in the case of ARP and uremic toxins. 
Although indoxyl sulfate and indole acetic acid, have basically similar structures, the binding constant for indoxyl sulfate is substantially greater than that for indole acetic acid. ${ }^{13)}$ These differences in binding constants can be attributed to the presence of a sulfate group on indoxyl sulfate and a carboxyl group on indole acetic acid. In fact, the binding constant for indoxyl sulfate and $p$-cresyl sulfate are nearly the same. ${ }^{14)}$ These results led us to the hypothesis that similar observations could be generated for the drugs binding to site II. However, this idea seems to be an oversimplification, because site II

Table 1. Primary Binding Parameters of Ligands of HSA at $\mathrm{pH} 7.4$ and $25^{\circ} \mathrm{C}$

\begin{tabular}{llcc}
\hline \hline Ligand & $n$ & $\mathrm{~K}\left(\times 10^{5} \mathrm{M}^{-1}\right)$ & Ref. \\
\hline Aripiprazole & 0.80 & 75.1 & Sakurama et al. $(8)^{a)}$ \\
Indole acetic acid & 1.0 & 2.1 & Sakai et al. $(14)^{a)}$ \\
Indoxyl sulfate & 1.0 & 16.1 & Sakai et al. $(14)^{a)}$ \\
& 1.0 & 0.98 & Watanabe et al. $(15)^{b)}$ \\
$p$-Cresyl sulfate & 0.80 & 12.1 & Watanabe et al. $(15)^{a)}$ \\
& 0.81 & 1.0 & Watanabe et al. $(15)^{b)}$ \\
\hline
\end{tabular}

a) $67 \mathrm{mM}$ phosphate buffer without $\mathrm{NaCl}$. b) $67 \mathrm{mM}$ phosphate buffer with $\mathrm{NaCl}$. consists of a diazepam subsite and an arylpropionic acid subsite. ${ }^{1)}$ We therefore examined the influence of the two toxins on the binding of diazepam and ibuprofen. As shown in Fig. 4 , the inhibitory effects of the toxins were significantly larger for diazepam, compared with that for ibuprofen. These results suggest that the site II drugs might be affected differently by the uremic toxins, depending on the location of the subsite site.

Induced CD Spectroscopy of Uremic Toxins Bound to HSA We also investigated the nature of the interaction of the uremic toxins with HSA using CD spectroscopy. On binding to HSA, indoxyl sulfate and indole acetic acid generated extrinsic Cotton effects with a positive sign in the $280-290 \mathrm{~nm}$ region. In contrast to the indole derivatives, negatively induced Cotton effects were observed in the $280-290 \mathrm{~nm}$ region for $p$-cresyl sulfate bound to HSA (Fig. 5).

Effect of Oleate on the Interaction between ARP and Indoxyl Sulfate HSA also functions as a fatty acid carrier in the blood and there are multiple binding sites on HSA for the binding of these molecules. In addition, it is known that oleic acid, a representative fatty acid, also binds to site II. Therefore, we investigated whether the presence of fatty acids affect to the binding of ARP to HSA in the presence or absence of
(A) Warfarin

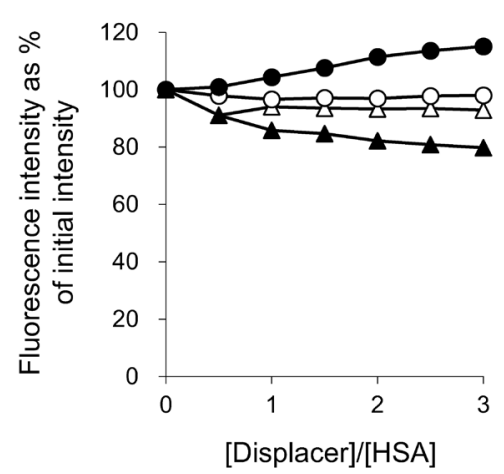

(B) Dansylsarcosine

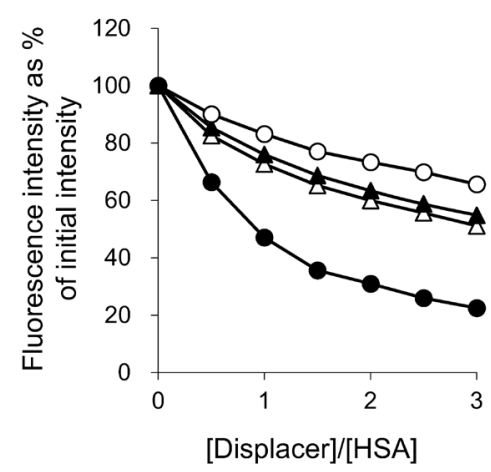

: ARP $\triangle$ : Indoxyl sulfate $\quad$ : Indole acetic acid $\Delta$ : $p$-Cresyl sulfate

Fig. 3. Fluorescent Intensity of Warfarin (A) and Dansylsarcosine (B) in the Presence of ARP or Uremic Toxins at $\mathrm{pH} 7.4$ and $25^{\circ} \mathrm{C}$

Fluorescent intensities of $2 \mu \mathrm{M}$ concentrations of warfarin and dansylsarcosine were measured in the presence of $2 \mu \mathrm{M}$ of HSA and each concentration $(0-6 \mu \mathrm{M})$ of $\mathrm{ARP}$ or uremic toxins respectively. The values are reported as the mean of three independent experiments.

(A) Diazepam

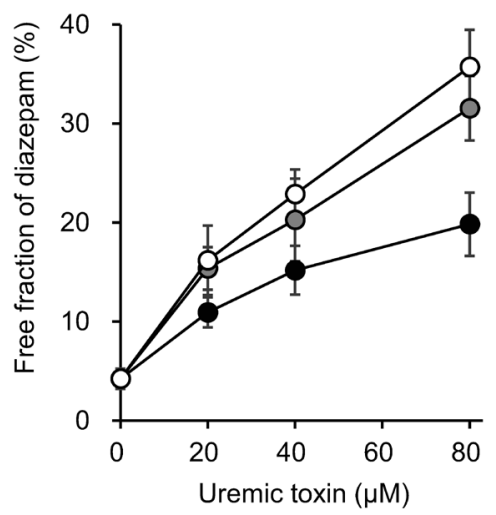

(B) Ibuprofen

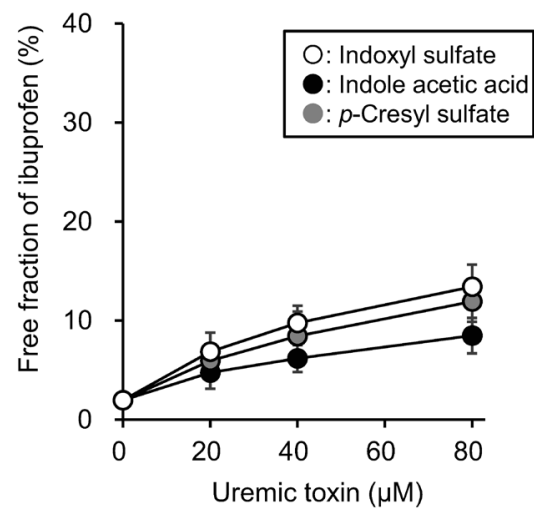

Fig. 4. Effects of Uremic Toxins on the Free Fractions of Diazepam (A) and Ibuprofen (B) at $\mathrm{pH} 7.4$ and $25^{\circ} \mathrm{C}$

The free fractions of diazepam or ibuprofen were measured in the presence of $20 \mu \mathrm{M}$ of diazepam or $20 \mu \mathrm{M}$ of ibuprofen and $40 \mu \mathrm{M}$ of HSA, and each concentration $(0-80 \mu \mathrm{M})$ of uremic toxins, respectively. The values are reported as the mean \pm S.D. of three independent experiments. 


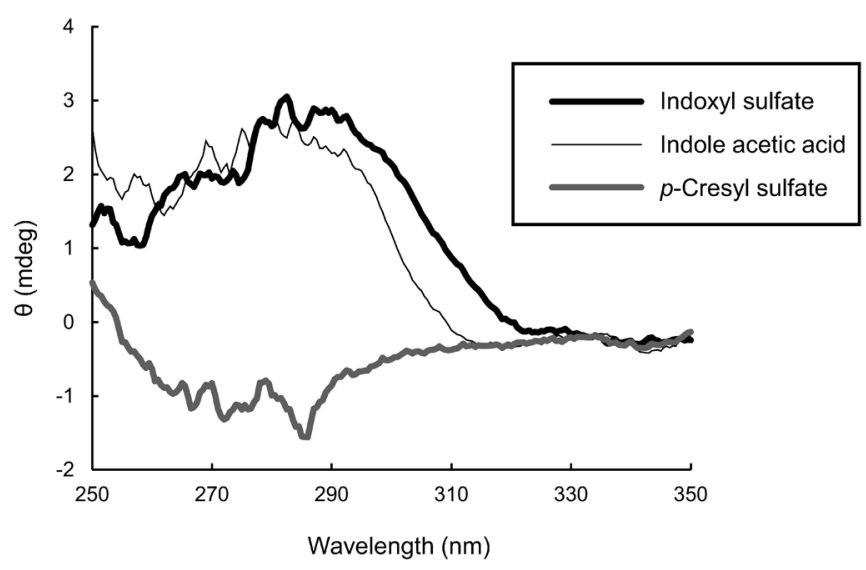

Fig. 5. Induced CD Spectra of Uremic Toxins with HSA at pH 7.4 and $25^{\circ} \mathrm{C}$

Each spectrum of the uremic substance is represented as the spectrum of the mixed solution of the uremic toxin and HSA minus the spectrum of HSA alone.

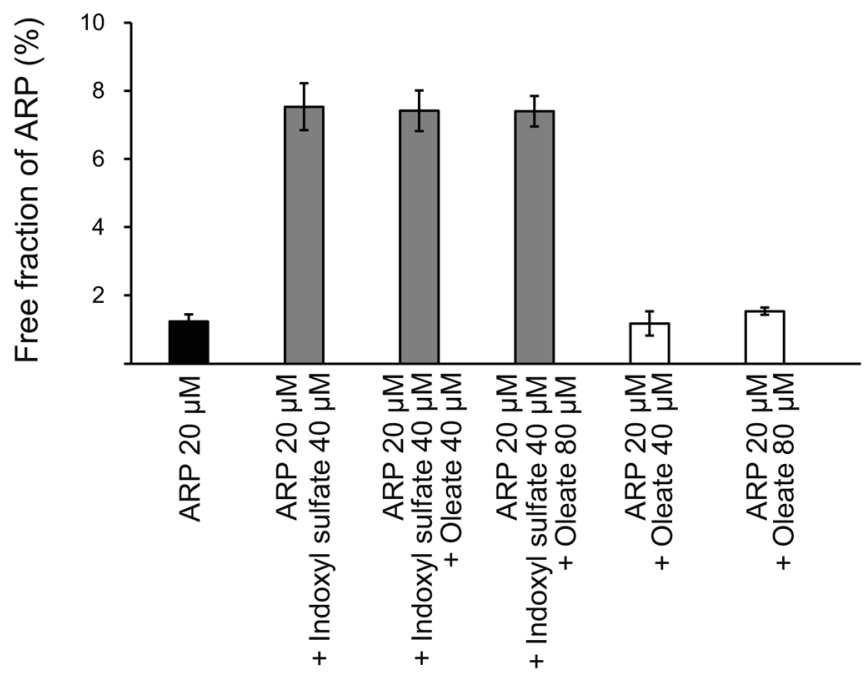

Fig. 6. Effect of Oleate on the Interaction between ARP and Indoxyl Sulfate at $\mathrm{pH} 7.4$ and $25^{\circ} \mathrm{C}$

The free fraction of ARP was measured in the presence of $20 \mu \mathrm{M}$ of ARP and $40 \mu \mathrm{M}$ of HSA, and $40 \mu \mathrm{M}$ of indoxyl sulfate or oleate. The values are reported as the mean \pm S.D. of three independent experiments.

indoxyl sulfate (Fig. 6). No significant effect of oleic acid on the binding of ARP to HSA in the presence or absence of indoxyl sulfate was observed.

\section{DISCUSSION}

Uremic toxins are well known as one of the major factors that inhibit the drug binding in serum. In patients with renal failure, indoxyl sulfate, indole acetic acid, and $p$-cresyl sulfate accumulate in the serum at concentrations as high as 44.5 , 2.4 and $41 \mathrm{mg} / \mathrm{L}$ (208.7, 13.7, and $217.9 \mu \mathrm{M}$ as the free form), respectively. ${ }^{12}$ The results of binding experiments indicated that these uremic toxins under renal disease level significantly inhibit the binding of ARP to HSA (Fig. 2), whereas uremic toxins under normal levels have no effect. To investigate the inhibition mechanism, we conducted a careful examination of the relationship between the binding sites of ARP and uremic toxins on HSA. The uremic toxins examined here primarily bound to site II on HSA. We focused on the interaction of the uremic toxins with ARP in site II. However, the inhibitory effects of ARP binding to HSA were clearly different (Fig. 2), depending on the chemical structures of the three uremic toxins. The differences in the inhibitory effects by uremic toxins can be explained by differences in the magnitude of the affinity to HSA. The opposite sign of the extrinsic Cotton effects for indoxyl sulfate and $p$-cresyl sulfate to HSA (Fig. 5) suggests that they have different orientations within the binding pocket. Induced Cotton effects are often observed when the electrons of a chromophore are perturbed by electrostatic forces associated with a nearby asymmetrical locus. ${ }^{15)}$ The sign of such an induced Cotton effect, whether extrinsic or intrinsic, is governed by the configuration of the asymmetrical center and its spatial relationship to the perturbed chromophore. ${ }^{16)}$ Schellman reported that the space around a chromophore may be divided into regions that make a positive or negative contribution to a Cotton effect. ${ }^{16)}$

Many patients with renal failure undergo hemodialysis to remove accumulated endogenous substances in the blood. Heparin, which is used as an anticoagulant in this treatment, is known to increase serum fatty acid levels. Increased fatty acid level frequently caused a change in drug binding to HSA. ${ }^{17)} \mathrm{We}$ previously found that the increased fatty acid concentrations by heparin triggered the phenomenon that led to increases in the concentration of the free form of a drug. ${ }^{18)}$ These findings prompted us to examine the effect of oleate, as a typical fatty acid, on the binding of ARP to HSA. Contrary to our expectations, oleate had no effect on the binding of the ARP-indoxyl sulfate system. This result can be explained by the differences in the binding site between oleate and indoxyl sulfate. In fact, in our previous binding experiments for a furosemide-CMPF (3carboxy-4-methyl-5-propyl-2-furanpropionate)-oleate system, we found a marked decrease in binding of furosemide. ${ }^{19)}$ The above ligands appear to bind to site I. That is, oleate may cobind to HSA along with the ARP molecule, rather than participate in a competitive interaction under these experimental concentrations. This result suggests that uremic toxins-fatty acids may stimulate the binding of ARP-HSA, but the effects would likely be smaller compared with drug-HSA interactions including furosemide-HSA under conditions of renal failure. We recently reported a 1.43-1.75-fold increase in the free fraction of ARP for oxidized HSA, which is a model of the HSA in patients with chronic renal failure, ${ }^{11)}$ compared to normal HSA. ${ }^{10)}$ In chronic renal failure, the oxidation of HSA becomes another factor that modifies the binding of ARP to HSA. Considering about a 6-fold increase in free fraction (from 1.23 to $7.53 \%$ ) in the presence of indoxyl sulfate (Fig. 6), the displacement of ARP from site II by uremic toxins may be more significant than that by oxidation. In a pathological situation such as chronic renal failure, the concentration of indoxyl acetic acid is lower than the values for indoxyl sulfate and $p$-cresyl sulfate. In addition, the binding constant of indoxyl acetic acid to HSA is lower than the values for indoxyl sulfate and $p$-cresyl sulfate. Therefore, indoxyl sulfate and $p$-cresyl sulfate could be major factors for the change in the binding of ARP to HSA in patients with chronic renal failure. In contrast, there is no information on changes in the pharmacokinetics of ARP during renal failure at this time. Hence, further detailed studies will be needed to clarify the effects of uremic toxins on the pharmacokinetics and pharmacodynamics of ARP in clinical situations. 
In conclusion, the data obtained in the present study will permit more relevant assessments of the pharmacokinetics and pharmacodynamics of ARP and related drugs that bind to site II on HSA, particularly drugs that bind to the diazepam subsite in site II during renal failure.

Conflict of Interest The authors declare no conflict of interest.

\section{REFERENCES}

1) Kragh-Hansen U, Chuang VT, Otagiri M. Practical aspects of the ligand-binding and enzymatic properties of human serum albumin. Biol. Pharm. Bull., 25, 695-704 (2002).

2) Yamasaki K, Chuang VTG, Maruyama T, Otagiri M. Albumin-drug interaction and its clinical implication. Biochim. Biophys. Acta, 1830, 5435-5443 (2013).

3) Gulyassy PF, Depner TA. Impaired binding of drugs and endogenous ligands in renal disease. Am. J. Kidney Dis., 2, 578-601 (1983)

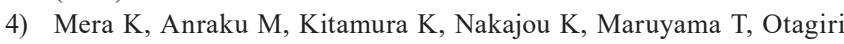
$\mathrm{M}$. The structure and function of oxidized albumin in hemodialysis patients: its role in elevated oxidative stress via neutrophil burst. Biochem. Biophys. Res. Commun., 334, 1322-1328 (2005).

5) Bowmer CJ, Lindup WE. Decreased drug binding in uraemia: effect of indoxyl sulphate and other endogenous substances on the binding of drugs of drugs and dyes to human serum albumin. Biochem. Pharmacol., 31, 319-323 (1982).

6) Lindup WE, Henderson SJ, Barker CE. Drug binding in renal disease. Plasma binding of drugs and its consequences. (Berpaire F, Bogaert M, Tillement JP, Verbeeck E, eds.) Academic Press, Waltham, pp. 103-120 (1991).

7) Ikeda K, Yoshitomi H, Nakayama T, Goto S, Kimura T. Plasma protein binding of furosemide in renal failure rabbits-investigation of endogenous protein binding inhibitors. J. Pharm. Pharmacol., 36, $663-667$ (1984).

8) Sakurama K, Kawai A, Tuan Giam Chuang V, Kanamori Y, Osa M, Taguchi K, Seo H, Maruyama T, Imoto S, Yamasaki K, Otagiri M. Analysis of the binding of aripiprazole to human serum albumin: the importance of a chloro-group in the chemical structure. $A C S$
Omega, 3, 13790-13797 (2018).

9) Sakurama K, Nishi K, Imoto $S$, Hashimoto M, Komatsu T, Morita Y, Taguchi K, Otagiri M, Yamasaki K. Further evidence regarding the importance role of chorine atoms of aripiprazole on binding to the site II area of human serum albumin. J. Pharm. Sci., 108, 1890-1895 (2019).

10) Enokida $T$, Yamasaki $K$, Okamoto $Y$, Taguchi $K$, Ishiguro $T$, Maruyama T, Seo H, Otagiri M. Tyrosine 411 and Arginine 410 of human serum albumin play an important role in the binding of sodium 4-phenylbutyrate to site II. J. Pharm. Sci., 105, 1987-1994 (2016).

11) Sakurama K, Nishi K, Chuang VTG, Hashimoto M, Yamasaki K, Otagiri M. Effects of oxidation of human serum albumin on the binding of aripiprazole. Biol. Pharm. Bull., 43, 1023-1026 (2020).

12) Duranton F, Cohen G, De Smet R, Rodriguez M, Jankowski J, Vanholder R, Argiles A. Normal and pathologic concentrations of uremic toxins. J. Am. Soc. Nephrol., 23, 1258-1270 (2012).

13) Sakai T, Takadate A, Otagiri M. Characterization of binding site of uremic toxins on human serum albumin. Biol. Pharm. Bull., 18, 1755-1761 (1995)

14) Watanabe H, Noguchi T, Miyamoto $Y$, Kadowaki D, Kotani S, Nakajima M, Miyamura S, Ishima Y, Otagiri M, Maruyama T. Interaction between two sulfate-conjugated uremic toxins, $p$-cresyl sufate and indoxyl sulfate, during binding with human serum albumin. Drug Metab. Dispos., 40, 1423-1428 (2012).

15) Chignell CF. Optical studies of drug-protein complexes IV: the interaction of warfarin and dicumarol with human serum albumin. Mol. Pharmacol., 6, 1-12 (1970).

16) Schellman JA. Symmetry rules for optical rotation. J. Chem. Phys., 44, 55 (1966).

17) Naranjo Ca, Sellers EM. Fatty acids modulation of drug binding to plasma proteins. Drug-protein binding. (Reidenberg MM, Erill S. eds.) Praeger Scientific, New York, pp. 233-251 (1986).

18) Sakai $T$, Maruyama $T$, Imamura H, Shimada H, Otagiri M. Mechanism of stereoselective serum binding of ketoprofen after hemodialysis. J. Pharmacol. Exp. Ther., 278, 786-792 (1996).

19) Takamura N, Maruyama T, Otagiri M. Effects of uremic toxins and fatty acids on serum protein binding of furosemide: possible mechanism of the binding defect in uremia. Clin. Chem., 43, 2274-2280 (1997). 